THÉoRÈme 1. I équivaut à II.

Démonstration. On a l'implication $I \rightarrow I I$. En effet, $H \subset \bar{R}$ et $R=\bar{R}-\Phi$ entraînent

\section{Division des régions partielles par les frontières et des frontières par les points}

par

\section{B. Knaster et J. Mioduszewski (Wrocław)}

Soient: $R$ une région, $\Phi=\operatorname{Fr}(R)$ sa frontière et $H$ une région partielle de $\bar{R}$, c'est-à-dire la partie commune et connexe d'un ensemble ouvert quelconque $G$ et de la fermeture de $R$ :

$$
H=G \cdot \bar{R} \text {. }
$$

Envisageons trois propriétés suivantes:

I. Tont ensemble fermé $F$ qui divise $R$ entre deux points $\left(^{1}\right)$ divise aussi $\bar{R}$ entre ces points.

II. La frontière $\Phi$ de $R$ ne divise ancune région partielle $H$ de $\bar{R}$. est situé.

ㄷ. Aucum point de $\Phi$ n'en divise la composante sur laquelle il

Tontes ces propriétés se présentent en particulier pour les régions planes $R$ qui sont des disques, c'est-à-dire dont les frontières $\Phi$ sont des courbes simples fermées. Par aillewrs, ces propriétés semblent de prime abord n'avoir rien de commun entre elles. Nous allons cependant établir leur équivalence dans des conditions bien générales, montrer par des exemples que ces conditions sont essentielles et noter des problèmes ouverts. Une des équivalences en question (théorème 1 qui suit) a été appliqué par l'un de nous (voir $[3]$, p. 315) dans la démonstration d'un théorème sur l'accessibilité de points (solution d'un problème de Wolibner); sinon, il $\mathrm{y}$ aurait fallu établir cette équivalence spécialement pour les disques.

L'exemple fort simple d'une région $R$ dépourvue des propriétés I-III à la fois est la partie du plan comprise entre deux circonférences tangentes intérieurement.

(1) Expressions synonymes: sépare deux points dans $R$, est un séparateur de $R$ (voir [2], p. 96).
(2) $\quad H-\Phi=H \cdot \bar{R}-\Phi=H \cdot(\bar{R}-\Phi)=H \cdot R=R-(\bar{R}-H)$.

$H$ divisé par $\Phi$ est donc la même chose que $R$ divisé par l'ensemble fermé $F=\bar{R}-H$, qui ne divise cependant pas $\bar{R}$, puisque

$$
\bar{R}-F=\bar{R}-(\bar{R}-H)=\bar{R} \cdot H=H
$$

et $H$ est connexe par définition.

On a l'implication IT $\rightarrow$ I. En effet, $R=\bar{R}-\Phi$ entraîne

$$
R-F=(\bar{R}-\Phi)-F=(\bar{R}-F)-\Phi \text {. }
$$

$R$ divisé par $F^{\prime}$ sans que $\bar{R}$ le soit est donc la même chose que la région partielle $H=\bar{R}-F$ divisée par la trontière $\Phi$.

THÉorìm 2. II équivaut à III pour toute région $R$ plane ant la frontière $\Phi$ est localement connexe.

Démonstration. On a l'implicatiou II $\rightarrow I I X$. En effet, supposons $q u$ 'il existe une composante de $\Phi$ divisée par un de ses points p. Par suite de la connexité locale de $\Phi$, il existe donc un arc $L \subset \Phi$ qui est divisé par $p$ en deux ares $L_{1}$ et $L_{2}$, situé chacun dans une autre composante de $\Phi-(p)$. Entourons $p$ d'un disque $D$ que $L$ divise en deux disques $D_{1}$ et $D_{2}$. On peut évidemment admettre que $L$ forme avec $\operatorname{Fr}(D)$ une courbe $\theta$, c'est-à-dire que $L \subset \bar{D}$ et que les bouts de $L$ seuls se trouvent sur $\operatorname{Fr}(D)$. La frontière $\Phi$ de $R$ étant localement connexe, le continu $\bar{R}$ l'est également (voir [2], p. 170,4). Soit $H$ la composante de $p$ dans $\bar{R} \cdot D$. En tant que composante d'un ensemble ouvert dans un continu localement connexe, $H$ est une région partielle de $\bar{R}$.

Reste à montrer que $\Phi$ divise $H$, à savoir que

$$
R \cdot D_{1} \cdot H \neq 0 \neq R \cdot D_{2} \cdot H
$$

La connexité locale de $\dot{\bar{R}}$ entraîne l'existence d'un disque $\Delta$ tel que $p \in \Delta, \bar{\Delta} C D$, que $p$ se laisse lier à tout point $q$ de $\bar{R} \cdot \Delta$ par un are $\widehat{p q} \subset \bar{R} \cdot D$, d'où

$$
\bar{R} \cdot \Delta \subset H
$$

et que $L$ divise $\Delta$ en deux disques

$$
\Delta_{1} \subset D_{1} \quad \text { et } \quad \Delta_{2} \subset D_{2}
$$

en formant avec $\operatorname{Fr}(\Delta)$ une courbe $\theta$. L'ensemble $L \cdot \operatorname{Fr}(\Delta)$ se compose done de deux points situés sur $L_{1}$ et $L_{2}$ respectivement (plus précisément 
sur $L_{1} \cdot \operatorname{Fr}\left(\Delta_{1}\right) \cdot \operatorname{Fr}\left(\Delta_{2}\right)$ et sur $L_{2} \cdot \operatorname{Fr}\left(\Delta_{1}\right) \cdot \operatorname{Fr}\left(\Delta_{2}\right)$ respectivement), donc dans deux composantes différentes de $\Phi-(p)$. On a $R \cdot\left(\Delta_{2}+\Delta_{2}\right) \neq 0$, car $p \in \bar{\Delta}=\overline{\Delta_{1}+\Delta_{2}}$ et $R$ est ouvert. On pent done admettre l'inégalité $R \cdot \Delta_{1} \neq 0$ (voir fig. 1 ).

Mais alors l'égalité $R \cdot \Delta_{2}=0$ est impossible, car elle aurait aussitôt pour conséquence que la frontière $\Phi$ de $R$ coupe ( $\left.{ }^{2}\right)$ le plan entre deux points quelconques $q_{1} \in R \cdot \Delta_{1}$ et $q_{2} \in \Delta_{2}-\bar{R}$. Par suite de la connexité locale de $\Phi$, il existerait done (voir [2], p. 361, 5) une courbe simple fermée $\Gamma \subset \Phi$ coupant le plan entre ces points. Le complément de $T$ au plan étant composé de deux disques, soit $\nabla$ celui qui contient $R$. Ancun des deux points de $L \cdot \operatorname{Fr}(\Delta)$ ne peut se trouver hors de $\bar{R}$, donc de $\bar{\nabla}$, car $L \subset \Phi \subset \bar{R}$ par définition. Les deux se trouvent donc dans $\bar{\nabla}=\nabla+\Gamma$. Cependant, si les deux étaient sur $\Gamma$, ils s'y laisseraient unir par un arc ne passant pas par $p$, ce qui est impossible, puisqu'on a $\Gamma \subset \Phi$ et les deux points en question appartiennent à des composantes différentes de $\Phi-(p)$. Par conséquent, au moins un point de $L \cdot \operatorname{Fr}(\Delta)$, donc de $L \cdot \operatorname{Fr}\left(\Delta_{2}\right)$ se trouve dans $\nabla$, d'où $\operatorname{Fr}\left(\Delta_{2}\right) \cdot \nabla \neq 0$. On a en même temps $\nabla-\Delta_{2} \neq 0$, car $\Delta_{2} \cdot \Delta_{1}=0$ et $R \cdot \Delta_{1} \neq 0$ entraînent $R-\Delta_{2} \neq 0$ et $\nabla$ contient $R$ par définition. Il en résulte ( $\left.{ }^{3}\right)$ que, réciproquement, $\operatorname{Fr}(\nabla) \cdot \Delta_{2}=\Gamma \cdot \Delta_{2} \neq 0$, d'où $\Phi \cdot \Delta_{2} \neq 0$, puisque $\Gamma \subset \Phi$. L'ensemble $\Delta_{2}$ étant ouvert, on a done finalement quand même $R \cdot \Delta_{2} \neq 0$.

Il est ainsi démontré que $R \cdot \Delta_{1} \neq 0 \neq R \cdot \Delta_{2}$. On a en outre $R \cdot \Delta_{1}$ $=R \cdot \Delta_{1} \cdot H$ et $R \cdot \Delta_{2}=R \cdot \Delta_{2} \cdot H$, puisque $\Delta_{1}+\Delta_{2} \subset \Delta$, d'où $R \cdot\left(\Delta_{1}+\Delta_{2}\right) \subset H$ en vertu de (4). Par conséquent, $R \cdot \Delta_{1} \cdot H \neq 0 \neq R \cdot \Delta_{2} \cdot H$, ce qui entraîne en vertu de (5) les inégalités (3) qu'il s'agissait d'établir.

On a l'implication III $\rightarrow$ II. En effet, $H$ étant ouvert dans $\bar{R}$, l'ensemble $\vec{R}-H$ y est fermé, done fermé tout court:

$$
\bar{R}-H=\overline{\bar{R}-H} \text {. }
$$

$H \subset \vec{R}$ entraîne $H=\bar{R}-(\bar{R}-H)=\bar{R}-\overline{\bar{R}-H}(\overline{R-(\bar{R}-H})=\overline{R \cdot \bar{H}}$, . d'où $H \subset H \cdot \vec{R} \cdot \vec{H}$. L'inclusion inverse étant triviale, on a l'égalité $H=\dot{H} \cdot \bar{R} \cdot \dot{H}$, qui peut être écrite dans la forme

\section{(7)}

$$
H=H \cdot \overline{H-\Phi}
$$

puisque $\boldsymbol{H} \cdot \boldsymbol{R}=H-\Phi$ d'après (2).

(2) Les notions de coupure et de division (séparation) coinneident pour les ensembles fermés dans les continus localement connexes (bornés ot non-bornés); voir [2], p. $187,8$.

(3) Nous appliquons ici le théorème tout à fait général suivant: $R_{1}$ et $R_{2}$ étant des régions, $\operatorname{Fr}\left(R_{1}\right) \cdot R_{\mathrm{z}} \neq 0$ et $R_{2}-R_{1} \neq 0$ éntraînent $\operatorname{Fr}\left(R_{2}\right) \cdot R_{1} \neq 0$. En effet, $R_{2}$ étant ouvert, la première inégalité enitraîne $R_{\mathrm{a}} \cdot R_{1} \neq 0$, d'où la troisièmé en vertu de la devarième, $R_{z}$, étant connexe:
Supposons, contrairement à II, que l'on ảit une décomposition

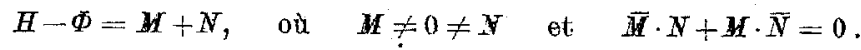

I s'ensuit en vertu de $(\bar{t})$ que $H=H \cdot(\overline{M+N})=\bar{M} \cdot H+\bar{N} \cdot H$, d'ok̀

$$
\bar{M} \cdot \bar{N} \cdot H \neq 0
$$

par suite de la connexité de $H$. On a en outre d'après (8)

d'où

$$
\bar{M} \cdot \bar{N} \cdot(H-\Phi)=\bar{M} \cdot \bar{N} \cdot(M+N)=0,
$$

$$
\vec{M} \cdot \bar{N} \cdot H \subset \Phi
$$

Soit, conformément à (9), $p$ un point quelconque de $\bar{M} \cdot \bar{N} \cdot H$. On a done $p \in \Phi$ en vertu de (10). L'ensemble fermé $\Phi$ étant localement connexe par hypothèse et $R$ étant par définition une composante de son complément au plan, l'ensemble $R+(p)$ est localement connexe (voir [2], p. 365,11 ). Connexe et topologiquement complet, il est done localement connexe par ares (voir même page et p. 184, 1). Par conséquent, $U$ étant un entourage ouvert de $p$, il existe dans $U$ un entourage ouvert $V$ de ce point, tel qu'en particnlier tout $q \in V \cdot H \cdot[R+(p)]$ se laisse unir à $p$ par un are $\widehat{p q} \subset U \cdot H \cdot[R+(p)]$. Les deux ensembles sont ouverts dans $H \cdot[R+(p)]$ et ils contiennent des points de $M$ et de $N$, car $p \in \bar{M} \cdot \bar{N} \cdot H$ et $M+N=H \cdot R$ d'après (2) et (8). Soient done $m \in M \cdot V$ et $n \in N \cdot V$. En posant $q=m$ et puis $q=n$, il existe par conséquent des ares $\overparen{m p}$ et $\overrightarrow{n p}$ dans $U \cdot H \cdot[R+(p)]$. Les ensembles $M \cdot U$ et $N \cdot U$ étant séparés en rertu de (8), on a nécessairement

$$
\widehat{m p} \subset M+(p) \quad \text { et } \quad \widehat{n p} \subset N+(p)
$$

Ainsi $A=\widehat{m p}+\widehat{p n}$ est un arc et $A \cdot \Phi=(p)$. Soit $B$ un arc quelconque unissant $m$ et $n$ dans $R$. On détermine d'une manière usuelle dans $A+B$ l'unique courbe simple fermée $C$ composée de deux arcs $A^{\prime} \subset A$ et $B^{\prime} \subset B$ unissant des points

$$
m^{\prime} \in M \cdot U \text { et } n^{\prime} \in N \cdot U
$$

et desquels l'are $A^{\prime}$ passe par $p$. Ainsi

$$
C \cdot \Phi=(p)
$$

Reste à montrer que, contrairement à III, la courbe $C$, donc le point $p$, divise la composante $\Phi^{\prime}$ de $\Phi$ sur laquelle $p$ est situé.

Fundamenta Mathematicae, T. XLV. 
Soient $D_{1}$ et $D_{2}$ les deux disques en lesquels $C$ coupe le plan. Comme $p \in H$ et $H$ est de la forme (1), il existe un disque $\Delta$ tel que $p \in \Delta$,

$$
\bar{\Delta} \subset \dot{G}
$$

et que l'intersection de $\operatorname{Fr}(\Delta)$ avec $O$ se compose exactement de deux points de $R$ :
(15)
$\operatorname{Fr}(\Delta) \cdot C=\left(m^{\prime \prime}\right)+\left(n^{\prime \prime}\right)$
où $\quad m^{\prime \prime} \in \widehat{m^{\prime} p}-(p)$
et $\quad u^{\prime \prime} \in \widehat{p^{\prime}}-(p)$

(voir (12) et fig. 2). Alor's la courbe $O$ divise $\Delta$ en deux disques:

$$
\triangle_{1} \subset D_{1} \text { et } \Delta_{2} \subset D_{2} \text {. }
$$

L'ensemble $\bar{\Delta}_{1}-\Phi$ ne contient aucun continu $L$ contenant les points $m^{\prime \prime}$ et $n^{\prime \prime}$, car on aurait en cas contraire d'une part $L \subset R$, ces points étant situés dans la région $R$ et $L$ étant par définition disjoint de la frontière $\Phi$ de cette région, et d'autre part $L \subset \overline{\Delta_{1}} \subset \bar{\Delta}$ entrainerait $L \subset G$ en vertu de (14). On aurait ainsi $L \subset R \cdot H=H-\Phi$ d'après (2), de sorte que les points $m^{\prime \prime}$ et $n^{\prime \prime}$ se trouveraient unis dans $H-\Phi$ par le continu $L$, ce qui est impossible en vertu de (8), puisque $m^{\prime \prime} \in M$ et $n^{\prime \prime} \in N$ d'après (11) et (15)

Il est ainsi établi que $\Phi$ est nne coupure de $\overline{\Delta_{1}}$ entre les points $m^{\prime \prime}$ et $n^{\prime \prime}$ de $\operatorname{Fr}\left(A_{1}\right)$. Il existe par conséquent (voir [2], p. 335, 1,336, et relativiser à $\overrightarrow{D_{1}}$ ) une composante $\Phi_{1}$ de $\Phi$ qui l'est également. En tant qu'un continu qui divise la fermeture de disque $\overline{\Delta_{1}}$ entre deux points $m^{\prime \prime}$ et $n^{\prime \prime}$ de la frontière $\operatorname{Fr}\left(\vec{A}_{1}\right)$, la, composante $\Phi_{1}$ a all moins un point commun avec l'are $\widehat{m^{\prime \prime} n^{\prime \prime}} C C$ de cette frontière et al moins un autre avec l'arc sans bouts $\operatorname{Fr}\left(\bar{A}_{1}\right)-\widehat{m^{\prime \prime} p n^{\prime \prime}}$. Par conséquent, on a d'une part $\Phi_{1} \cdot C \neq 0$, d'où $p \in \Phi_{1}$, puisque $\Phi_{1} \cdot C \subset \Phi \cdot O=(p)$ d'après (13), et d'autre part $\Phi_{1} \cdot D_{1} \neq 0$, puisque $\operatorname{Fr}\left(\Delta_{1}\right)-C C D_{1}$ conformément à (16).

On établit pareillement l'existence d'une composante $\Phi_{2}$ de $\Phi$ qui divise $\bar{A}_{2}$ entre $m^{\prime \prime}$ et $n^{\prime \prime}$ et pour laquelle on a $p \in \Phi_{2}$ et $\Phi_{2} \cdot D_{2} \neq 0$.

Nous conchons: les composantes $\Phi_{1}$ et $\Phi_{2}$ ne constituent qu'une seule composante, puisqu'elles ont un point commun. Désignons-la par $\Phi^{\prime}$. Ayant des points communs avec les deux régions $D_{1}$ et $D_{2}$ en lesquelles la courbe $C$ coupe le plan, $\Phi^{\prime}$ est coupée par $C$, done par le point $p$ en vertu de (13). Ce point la divise, la connexité locale de $\Phi$ entraînant celle de ses composantes (voir aussi le renvoi $\left({ }^{2}\right)$, p. 308).

Le théorème 2 étant ainsi démontré, envisageons-en les hypothèses.

Celle que la région $R$ soit située sur le plan est essentielle (si l'on ne compte pas la droite, où théorème est trivial). L'exemple dans lequel $R$ est la partie de l'espace comprise entre deux surfaces sphériques tangentes intérieurement montre que l'hypothèse de $n=2$ est essentielle pour l'implication II $\rightarrow$ III. Afin de montrer qu'elle l'est également pour l'implication inverse, soit $R$ la région bornée par la surface du demi-tore $\left(1 \overline{x^{2}+z^{2}}-2\right)^{2}+y^{2} \leqslant 1, z \leqslant 0$ et par celle de deux cônes ayant les cercles $(x \pm 2)^{2}+y^{2} \leqslant 1$ pour bases et le point $(0,0,2)$ pour sommet commun. La frontière $\Phi$ de $R$ coupe la région partielle $H$ de $\bar{R}$ composée de deux cônes sans bases (puisque déjà leur sommet la coupe). Cependant, aucun point ne coupe $\Phi$. Des exemples analogues peuvent être indiqués facilement dans les espaces euclidiens à $x>3$ dimensions.

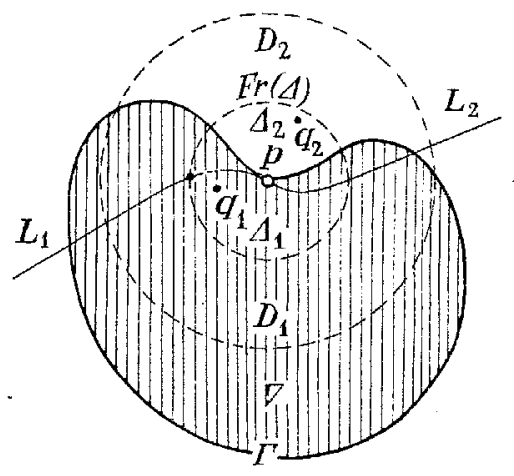

Fig. 1

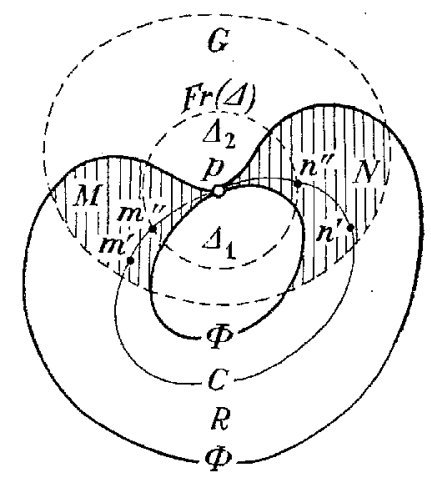

Fin. 2
L'bypothèse de la connexité locale de la frontière $\Phi$ de $R$ est également essentielle. L'exemple suivant, dû à A. Lelek, le montre pour I'implication $I \rightarrow I I$. Soient sur le plan Oxy: $D$ le cercle ouvert $\varrho<1$ et $S$ la spirale $\varrho=\varphi /(\pi+\varphi)$ qui en approche indéfiniment la circonférence ( $\varrho$ désignant la distance entre le point $(\varrho, \varphi)$ et l'origine $O$, et $\varphi$ désignant l'angle compté de 0 à $+\infty$ à partir de l'axe $O_{x}$ ). Considérons les ares de cette spirale, disjoints deux à deux,

$$
S_{n}=E_{(\varrho, q) \in S}[6 n \pi \leqslant \varphi \leqslant(6 n+4) \pi] \text { où } \quad n=0, \mathbf{1}, \ldots,
$$

entourons-les de cercles généralisés fermés, encore disjoints deux à denx

$$
Q_{n}=\sum_{p \in S_{n}} E\left[|p-q| \leqslant 1 / 54(n+1)^{2}\right]
$$

(| | désignant ici la distance) et posons

$$
P=D-\sum_{n=0}^{\infty} Q_{n} \text {. }
$$


Ainsi défini, $P$ est une région (et même un domaine ouvert, à savoir I'intérieur du continu $\vec{P})$, sa frontière $\operatorname{Er}(P)$ n'est pas localement connexe, à savoir aux points de $\operatorname{Fr}(D)$, elle a $x_{0}$ composantes, à sàvöir "les ' courbes simples fermées $\operatorname{Fr}\left(Q_{n}\right)$ et la circonférence $\operatorname{Fr}(D)$, non-dense dans Fr $(P)$. L'identification des points diamétralement opposés $(1, \pi / 2)$ et $(1,3 \pi / 2)$ de $\operatorname{Fr}(D)$ transforme $\bar{P}$ en une image homéomorphhe du continu plan qui est borné par une courbe $C$ composée de deux circonférences tangentes intérieurement au point $p$ (image des points identifiés) et par une infinité de courbes simples fermées situées entre elles. L'image $R$ de $P$ est une région (en même temps qu'un domaine onvert), sa frontière $\Phi$ (l'image de celle de $P$ ) ne divise aucune région partielle du continu $\bar{R}$ et le point $p$ divise la composante $C$ de $\Phi$. Or $\Phi$ n'est localement connexe en aucun point de. $C$.

La connexité locale de $\Phi$ est essentielle aussi pour l'implication III $\rightarrow$ II: il n'y suffit même pas que $\bar{R}$ soit localement connexe. Soit d'abord $\Phi$ le continu formé par la circonférence $\left(x-\frac{1}{2}\right)^{2}+y^{2}=1$ et par le continu indécomposable $\mathscr{B}$ (voir $[1]$, p. 40 ), ayant avec elle la demi-circonférence supérieure commune. $R$ étant la régionb ornée par $\Phi$, le continu $\bar{R}$ est le eercle borné par la circonférence en question .Soit $H$ le cercle ouvert $\left(x-\frac{1}{2}\right)^{2}+y^{2}<\frac{1}{16}$. C'est done bien une région partielle de $\bar{R}$. Elle est divisée par $\mathscr{B}$, done à plus forte raison par $\Phi$. Cependant, aucun point ne divise $\Phi$.

Dans cet exemple, la région $R$ n'est pas un domaine ouvert. Nous ajoutons donc ici un autre où $R$ l'est et que nouss croyons d'ailleurs intéressant plutôt pour d'autres raisons topologiqnes qu'à cause du détail si peu important par lui même. Ii'exemple en question a été également imaginé par A. Lelek.

Soient: $\mathcal{C}$ l'ènsemble parfait non-dense de Cantor sur le segment $0 \leqslant x \leqslant 1$ de l'axe d'abscisses, $I_{i j}$ où $i=1,2, \ldots$ et $j=1,2, \ldots, 2^{i-1}$, le segment $a_{i j} \leqslant x \leqslant b_{i j}$ contigu ̀̀ $\mathcal{C}$ (fermeture du $j$-ième intervalle contigu de longueur $1 / 3^{i}$ ), $I$ le segment $0 \leqslant y \leqslant \frac{1}{3}$ de l'axe d'ordonnées, $D_{i j k}$ où $k=1,2, \ldots, 3^{i-1}$ celui de denx demi-disques circulaires fermés de centre $\left(a_{i j},\left(k-\frac{1}{2}\right) / 3^{i}\right)$ et de rayon $1 / 2: 3^{i}$ qui fait partie du rectangle $J_{i j}=I_{i j} \times I$, Q l'intérieur du carré aux sommets opposés $(0,0)$ et $(1,1)$, enfin .

$$
R=Q-\overline{\sum_{i=1}^{\infty} \sum_{i=1}^{i^{i-1}} \sum_{i=1}^{j^{i-1}} D_{i j k} .}
$$

La soustraction n'intéresse done que le tiers inférieur de $Q$. $R$ est une région (et un domaine ouvert), le continu $\bar{R}$ est localement connexe, sa frontière $\Phi$, qui est d'ailleurs aussi continu, ne l'est pas au point. $\left(\theta, \frac{1}{3}\right)$ par exemple), aucun point ne dipise $\Phi$ (bien que tout point $p \in O \times I$ le coupe) et enfin $\Phi$ divise la région partielle $H=G \cdot \bar{R}$ de $\bar{R}$ où $G$ est l'ensemble des points du plan d'ordonnée inférieure à̀ $\frac{1}{3}$.

Probleimes. Est-ce que la propriété III avec le mot, ,sous-continu a au lieu du mot „point" entraîne la propriété II sur le plan, en particulier lorsque $\Phi$ n'est pas localement connexe?

Est-ce que tont continu $\Phi$ qui n'est pas localement connexe, mais qui est la frontière d'une région plane $R$, contient un point qui le coupe (au sens de [2], p. 129)?

Est que la propriété III équivaut, et dans quelles conditions, à la suivante: aucun point ne divise $\Phi$ localement en plus de 2 composantes?

\section{Travaux cites}

[1] B. Knaster et C. Kuratowski, Sur les continus non bornés, Fundamenta Mathematicae 5 (1924), p. 23-58.

[2] C. Kuratowski, Topologie II, Monografie Matematyczne, tome XXI, Warszawa-Wrockaw 1950.

[3] J. Mioduszewski, Sur l'aceessibilité des points d'ensembles fermés dans les espaces euelidiens, Fundamenta Mathematicae, ce volume, p. 314-319.

INSTYTUT MATEMATYCZNY UNIWERSYTETU WROCEAWSKIEGO INSTITUT MATHEMATIQUE DE L'UNIVERSITE DE WROCEAW

Reģu par la Rédaction le 13.8.1957 